Arq. Bras. Med. Vet. Zootec., v.64,n.5, p.1094-1100, 2012

\title{
Estudo clínico, histopatológico e imunoistoquímico de neoplasias mamárias em cadelas
}

\author{
[Clinical, histopathological and immunohistochemical study of mammary \\ neoplasm in bitches] \\ M.A.R. Feliciano, A.S. Silva, R.V.R. Peixoto, P.D. Galera, W.R.R. Vicente \\ Faculdade de Ciências Agrárias e Veterinárias - UNESP - Jaboticabal, SP
}

\section{RESUMO}

O objetivo foi realizar um estudo clínico e epidemiológico de neoplasias mamárias em cadelas, considerando-se histórico reprodutivo, exame físico, diagnóstico histopatológico e imunoistoquímico. Utilizaram-se 60 neoplasias mamárias, divididas em grupos (grupo 1 - benigno, e grupo 2 - maligno). Avaliaram-se dados do histórico reprodutivo, o exame físico e achados histopatológicos e imunoistoquímicos para fator de crescimento endotelial vascular. Ao estudo do histórico reprodutivo, encontraram-se $90 \%$ dos animais com irregularidade de cio, 86,63\% das cadelas não foram medicadas com contraceptivos e 83,33\% não eram castradas. Ao exame físico, não foi verificada diferença $(p>0,05)$ entre grupos ao se avaliar consistência das massas, regularidade da superfície tumoral e localização anatômica dos tumores. Quanto ao tamanho das massas, verificou-se diferença entre os grupos $(\mathrm{p}=0,0077)$, com $0,78 \pm 1,13 \mathrm{~cm}$ para o grupo 1 e $1,81 \pm 2,29 \mathrm{~cm}$ para o grupo 2 . Diagnosticaram-se $40 \% \mathrm{de}$ massas benignas e $60 \%$ de malignas, de acordo com os tipos de neoplasias. Para VEGF, verificaram-se

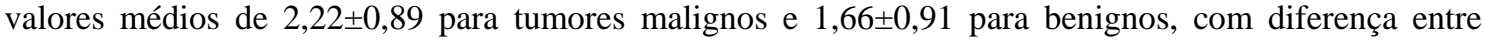
grupos $(\mathrm{p}=0,0315)$. As neoplasias mamárias em cadelas não apresentam características de histórico reprodutivo e de exame clínico que auxiliem o diagnóstico diferencial, sendo a histopatologia o único método para conclusão do diagnóstico e a imunoistoquímica podendo ser utilizada para prognóstico da lesão.

Palavras-chave: cadelas, neoplasias mamárias, estudo clínico, histopatologia

\begin{abstract}
The objective was to conduct a clinical and epidemiological study of mammary cancer in bitches, considering their reproductive history, physical examination, histopathological and immunohistochemical diagnosis. We used 60 breast tumors which were divided into groups (group 1 group 2 and benign - malignant). We evaluated data from the reproductive history, physical examination and histopathology and immunohistochemistry for VEGF. The study of reproductive history had $90 \%$ of irregular estrus, $86.63 \%$ of the dogs were not tested with contraceptives and $83.33 \%$ were not castrated. On physical examination, there was no difference $(p>0.05)$ between groups regarding the consistency of the masses, surface regularity of the tumor and anatomic location of tumors. As for the masses, there was a difference between groups $(p=0.0077)$, with $0.78 \pm 1.13 \mathrm{~cm}$ for group 1 and $1.81 \pm 2.29 \mathrm{~cm}$ for group 2 . $40 \%$ of benign masses and 60\% of malignant masses were diagnosed, according to the types of malignancies. For VEGF, the average values were $2.22 \pm 0.89$ for malignant tumors and $1.66 \pm 0.91$ for benign, with differences between groups $(p=0.0315)$. The mammary tumors do not exhibit characteristics of reproductive history and clinical examination to help the differential diagnosis, and histopathology is the only method for completion of diagnosis and immunohistochemistry which can be used for injury prognosis.
\end{abstract}

Keywords: bitches, mammary neoplasms, clinical study, histopathology

Recebido em 2 de março de 2011

Aceito em 20 de julho de 2012

E-mail: marcusfeliciano@yahoo.com.br 


\section{INTRODUÇÃO}

A neoplasia mamária em cadela apresenta significativa importância em medicina veterinária e tem sido muito investigada, principalmente, por servir de modelo para o estudo do câncer de mama na mulher (Martins e Ferreira, 2003). Os tumores mamários dos caninos apresentam várias características epidemiológicas, clínicas, biológicas e genéticas semelhantes aos da espécie humana. Entre estas, podem ser citados: faixa etária de aparecimento, morfologia, efeito protetor da ováriohisterectomia, presença de receptores de estrógeno e progesterona, órgãos alvo de metástase, evolução clínica e hereditariedade em alguns casos. Também foi demonstrado que neoplasias mamárias de cadelas apresentam fenótipo antigênico comparável àquele observado em lesões de mama em seres humanos com homologia entre ambos os genes (Silva et al., 2004).

As neoplasias mamárias dos canídeos são modelos apropriados e válidos no estudo da biologia do câncer nesta espécie e em seres humanos, assim como para testes de agentes terapêuticos, já que animais de companhia têm tumores que, do ponto de vista histopatológico e de comportamento biológico, são similares aos descritos na espécie humana (Zuccari et al., 2004).

Os tumores mamários caninos constituem, aproximadamente, $52 \%$ de todas as neoplasias que afetam as fêmeas desta espécie, com cerca de $50 \%$ dos tumores apresentando características de malignidade (Queiroga e Lopes, 2002). Cerca de $50 \%$ dos casos clínicos de neoplasias mamárias em cadelas aparecem em consulta, apresentando lesões múltiplas, não apenas devido à rapidez de progressão do tumor, mas também devido ao adiamento na apresentação dos animais à avaliação clínica do médico veterinário (Lana et al., 2007).

A primeira abordagem de paciente com nódulos mamários deve consistir em exame físico minucioso, não apenas das glândulas mamárias, mas também de características gerais que possibilitem avaliar o estado geral do animal. Ao exame físico da cadeia mamária, devem ser registrados os seguintes aspectos das lesões: número, localização, consistência e tamanho, assim como eventuais sinais de aderência aos tecidos adjacentes, deformações das mamas e ulceração na pele (Lana et al., 2007).

O diagnóstico diferencial é possível por meio da avaliação microscópica, por citologia ou histopatologia (Misdrop et al., 1999). Segundo estes autores, os diagnósticos diferenciais que devem ser considerados na apresentação clínica de nódulos mamários são: 1) tumores mamários malignos; 2) tumores mamários benignos; 3) tumores cutâneos e subcutâneos; 4) mastite; e 5) hiperplasias/displasias mamárias.

Histologicamente, os tumores mamários são interpretados como benignos ou malignos. Com base no histórico do animal e no seu exame físico, o tumor maligno caracteriza-se por apresentar crescimento rápido, infiltração nos tecidos vizinhos e frequente ulceração. O tumor benigno possui crescimento lento e expansivo, tendo área circunscrita, não aderindo aos tecidos adjacentes; suas células são bem diferenciadas, próximas do tecido normal, e as atipias celulares são raras e discretas (Carvalho, 2006).

Os tumores malignos têm, caracteristicamente, a capacidade de induzir neoformação vascular e, ao mesmo tempo em que aumentam de tamanho, estimulam esta neovascularização pela secreção de fatores angiogênicos (Lucena, 2006).

$O$ fator de crescimento endotelial vascular (VEGF) é uma proteína dimérica que estimula a migração e a proliferação de células endoteliais vasculares. A proliferação celular induzida por VEGF promove metástase: 1) induzindo a formação da neovascularização, 2) produzindo colagenase e outras enzimas de degradação que facilitam a proliferação de células neoplásicas e 3) estimulando as células neoplásicas (Restucci et al., 2002).

No trabalho de Graham e Myers (1999), foi demonstrado que a angiogênese pode ser um indicador independente e significativo do prognóstico nas neoplasias malignas de mama em cadelas, assim como é na mulher. O VEGF é mais comumente estudado em oncologia humana, sendo poucos os estudos em medicina veterinária (Restucci et al., 2002).

O presente trabalho tem como objetivo demonstrar os resultados de um estudo clínico e 
epidemiológico de neoplasias mamárias de cadelas, considerando os dados do histórico reprodutivo dos animais, do exame físico e dos diagnósticos histopatológico e imunoistoquímico.

\section{MATERIAL E MÉTODOS}

Foram utilizadas 60 cadelas, de diferentes idades e raças, pacientes do Setor de Obstetrícia da UNESP/Jaboticabal. Todo o procedimento foi aprovado pela Comissão de Ética no Uso de Animais, processo no. 018568-08, UNESP/Jaboticabal.

Os animais foram submetidos aos exames clínico e radiográfico do tórax e ao hemograma. Esses resultados foram importantes para determinar a história reprodutiva do animal, a evolução da massa mamária, a avaliação do estado de saúde da paciente e para a caracterização da afecção mamária, visando ao encaminhamento à mastectomia.

Foram utilizadas 60 neoplasias mamárias. Após a realização do estudo clínico das neoplasias, os animais foram divididos em dois grupos experimentais, segundo as características histopatológicas (Misdrop et al., 1999):

grupo 1: neoplasia mamária com características benignas $(n=24)$;

grupo 2: neoplasia mamária com características malignas $(\mathrm{n}=36)$.

A realização da radiografia torácica foi indicada para diagnóstico de metástase pulmonar e avaliação cardíaca, antes do procedimento cirúrgico.

Todos os dados obtidos na avaliação prévia foram arquivados junto com a identificação do animal.

O histórico reprodutivo dos animais foi abordado durante a realização da anamnese, buscando-se informações referentes à esterilidade dos animais (castrados ou não), à administração de contraceptivos e quanto à regularidade do estro das cadelas.
Ao exame físico das neoplasias, foram determinadas suas características macroscópicas segundo a evolução tumoral (lenta ou rápida), a regularidade da superfície (regular ou irregular), a consistência da massa (firme ou flutuante), o tamanho (centímetros) e a localização anatômica tumoral. O tamanho das massas foi obtido com auxílio de paquímetro, sendo realizada a medida de seu diâmetro.

Fragmentos dos tumores mamários foram colhidos após mastectomia, e considerando-se as dimensões do tumor e sua relação com o tecido adjacente, foram encaminhados para processamento e diagnóstico histopatológico.

O aspecto macroscópico de cada neoplasia foi avaliado, e os fragmentos colhidos foram fixados em solução de formol a $10 \%$, tamponado com fosfatos $(\mathrm{pH} \mathrm{7,4)} \mathrm{e} \mathrm{processados} \mathrm{rotineiramente}$ até a inclusão em parafina. Cortes de $5 \mu \mathrm{m}$ foram feitos em micrótomo e posteriormente corados por hematoxilina e eosina. Em análise com microscopia de luz, efetuou-se a classificação da neoplasia, segundo os critérios da Organização Mundial da Saúde (Misdrop et al., 1999).

A técnica de imunoistoquímica, utilizando-se kit polimerase (NovoLink - TM Polymer Detection System) e anticorpo para VEGF (Abcam Mouse monoclonal VG-1-VGEF), foi aplicada para se avaliar a expressão dos antígenos tumorais (Turley et al., 1998; Rebêlo, 2009).

A imunorreação foi analisada de forma semiquantitativa, segundo a intensidade da coloração acastanhada no citoplasma das células neoplásicas, usando-se os seguintes escores: 1=ausência de coloração; 2=coloração fraca; $3=$ coloração média e 4=coloração forte (Turley et al., 1998) (Fig. 1). Os valores de VEGF foram correlacionados com variáveis do modo Doppler para vascularização.

O delineamento experimental foi inteiramente casualizado. Os dados foram previamente testados para normalidade dos resíduos e homogeneidade das variâncias (teste F). Foram utilizados o PROC MEANS-SAS ${ }^{\circledR}$ e $\quad$ o GraphPad Prisma 4. 

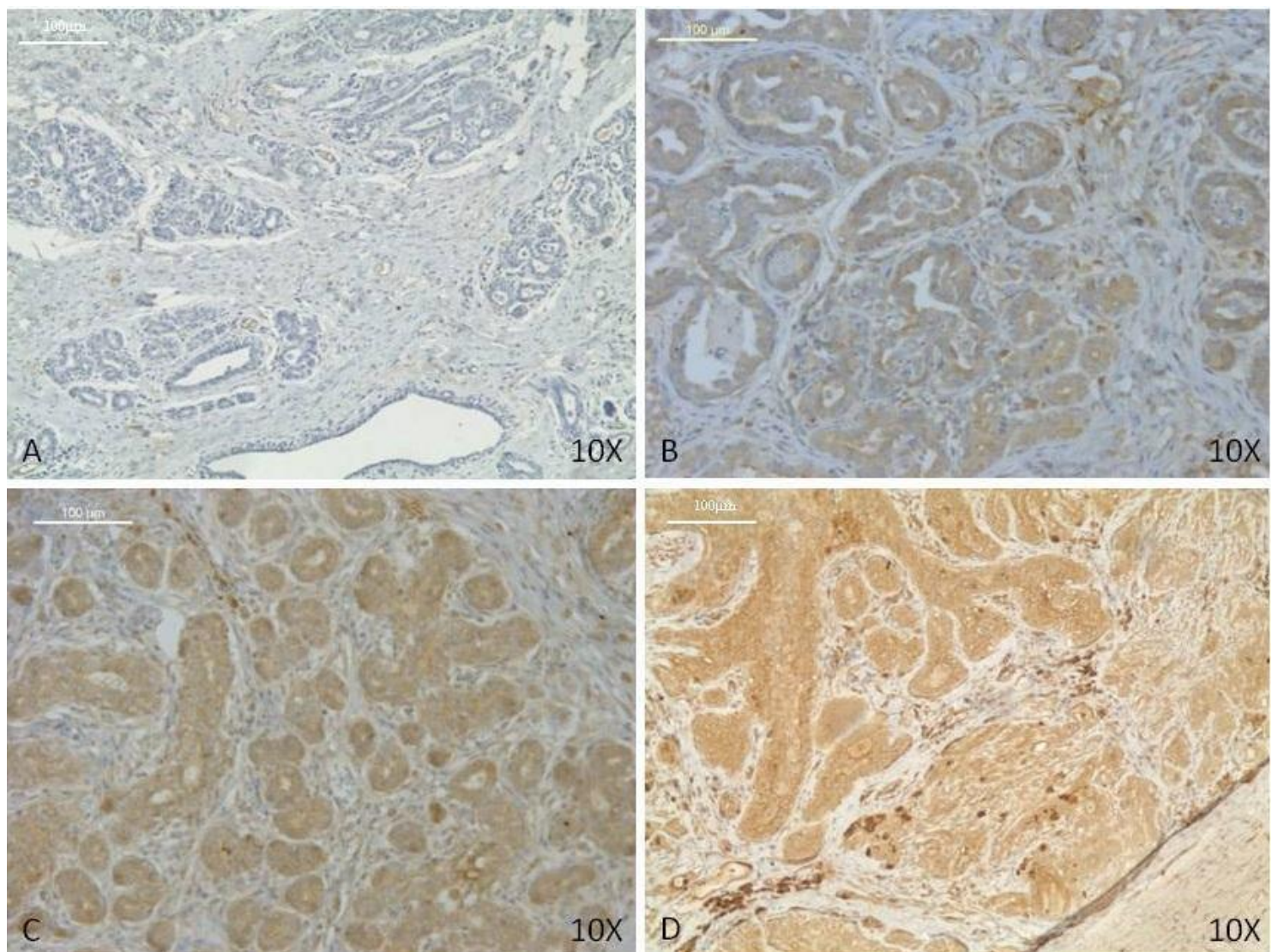

Figura 1. Fotomicrografias de neoplasias mamárias em cadelas com imunoistoquímica para fator de crescimento endotelial vascular (VEGF). Classificação semiquantitativa: (A) escore 1 - coloração ausente; (B) escore 2 - coloração fraca; (C) escore 3 - coloração moderada; (D) escore 4 - coloração forte.

As médias reais ou transformadas foram avaliadas pela análise de variância e, quando com significância, foi realizado o desdobramento do estudo estatístico de médias pelo teste pareado de médias (teste t). As médias não homogêneas em teste de normalidade foram avaliadas por meio do teste Mann-Whitney. As porcentagens e frequências foram avaliadas por meio dos testes qui-quadrado e exato de Fischer. A correlação de dados experimentais foi realizada por meio de regressão linear. Foi utilizado nível de significância de 5\% para todos os testes realizados.

\section{RESULTADOS E DISCUSSÃO}

As cadelas não apresentaram alterações hematológicas e radiográficas, representadas por metástase pulmonar, que pudessem impedir a realização dos procedimentos de colheita das neoplasias por meio da mastectomia.
Mediante a anamnese, obtiveram-se dados relevantes sobre regularidade do estro, administração de anticoncepcionais e castração. Cerca de $10 \%$ das cadelas apresentavam regularidade do estro, enquanto $90 \%$ tinham irregularidade, em sua maior parte em intervalos curtos. Das cadelas, 13,37\% haviam sido tratadas com contraceptivos, segundo informação dos proprietários. Em relação à castração, somente $16,67 \%$ das cadelas eram ovário-histerectomizadas. Os resultados assemelham-se aos de Queiroga e Lopes (2002), que encontraram menor incidência de neoplasias mamárias em cadelas castradas. Cadelas que apresentam estro com intervalos irregulares e que receberam administração de contraceptivos apresentam grande probabilidade de manifestarem neoplasias mamárias, devido aos fatores hormonais relacionados com a fisiopatologia da síndrome (Queiroga e Lopes 2002). Nesse estudo, pôde-se observar baixa 
ocorrência de cadelas com neoplasia mamária que receberam drogas contraceptivas e que apresentavam estro com intervalos regulares, demonstrando o acometimento de tumores em cadelas sem alterações reprodutivas.

Ao exame específico, em 100\% das neoplasias do grupo 1 e $92 \%$ do grupo 2 verificou-se consistência firme, com apenas três neoplasias malignas apresentando consistência flutuante. Para a consistência tumoral, não foi encontrada diferença significativa entre os grupos experimentais $(\mathrm{p}=0,2678)$.

Para a característica de regularidade da superfície tumoral, não se observou diferença entre os grupos experimentais $(\mathrm{p}=0.1006)$. Encontrou-se presença de regularidade na superfície de $92 \%$ das neoplasias benignas e $75 \%$ das malignas. Oito por cento das amostras do grupo 1 e $25 \%$ do grupo 2 apresentaram superfície irregular.

Ao se avaliar o tamanho das neoplasias mamárias, entre os grupos experimentais, verificou-se diferença $(\mathrm{p}=0,0077)$. As massas do grupo 1 apresentaram um tamanho médio de $0,78 \pm 1,13 \mathrm{~cm}$, e as do grupo 2 de $1,81 \pm 2,29 \mathrm{~cm}$.

Não houve diferença entre os grupos experimentais em relação à localização anatômica das neoplasias mamárias $(\mathrm{p}=0,1404)$, como demonstrado na Tab. 1.

Tabela 1. Localização anatômica de neoplasias mamárias em cadelas, examinadas no Setor de Obstetrícia da UNESP/Jaboticabal

\begin{tabular}{|c|c|c|c|c|c|}
\hline Antímero esquerdo & I1 & $\mathrm{A} 2$ & A1 & $\mathrm{T} 2$ & $\mathrm{~T} 1$ \\
\hline Grupo 1 & 5 & 2 & 3 & 1 & 1 \\
\hline Grupo 2 & 9 & 4 & 11 & 0 & 0 \\
\hline Total & 14 & 6 & 14 & 1 & 1 \\
\hline Antímero direito & I1 & $\mathrm{A} 2$ & A1 & $\mathrm{T} 2$ & $\mathrm{~T} 1$ \\
\hline Grupo 1 & 2 & 5 & 2 & 1 & 2 \\
\hline Grupo 2 & 4 & 1 & 4 & 3 & 0 \\
\hline Total & 6 & 6 & 6 & 4 & 2 \\
\hline
\end{tabular}

I1: mama inguinal; A2: mama abdominal caudal; A1: mama abdominal cranial; T2: mama torácica caudal; T1: mama torácica cranial.

Tabela de contingência para teste de qui-quadrado em nível de significância de 5\%.

A consistência tumoral observada no presente estudo não está diretamente relacionada ao tipo de tumor de mama encontrado; entretanto, sugere-se que a presença de estruturas firmes ou macias pode estar correlacionada com a presença ou não de áreas císticas na neoplasia. Em tumores de consistência flutuante, as lesões císticas são relevantes para a caracterização da homogeneidade do parênquima tumoral, característica citada por Bastan et al. (2009) como importante na diferenciação tumoral. Todavia, neste experimento não houve diferença $(p>0,05)$ entre os tecidos tumorais avaliados com o tipo de consistência tumoral, sendo que a maioria apresentou característica firme à inspeção.

Quanto à regularidade da superfície tumoral, a maioria das neoplasias apresentou margens regulares, o que difere do encontrado por Misdrop et al. (1999) e Lana et al. (2007), que consideram a presença de irregularidade da superfície tumoral como um forte indicativo para ocorrência de neoplasias malignas.

Em relação ao tamanho tumoral, Bastan et al. (2009) e Nyman et al. (2006) encontraram diferença significativa entre tumores mamários malignos e benignos, o que se assemelha aos resultados encontrados, demonstrando que as neoplasias mamárias malignas apresentaram tamanhos maiores que as benignas. Nyman et al. (2006) comentam que tumores maiores podem ser decorrência da presença de alterações do parênquima, relacionadas com o grau de malignidade.

Os resultados mostram maior incidência de tumores mamários em cadeia inguinal, entretanto não houve correlação significativa da localização tumoral com o tipo de tumor $(\mathrm{p}=0,1404)$, o que 
difere do citado por Lana et al. (2007), os quais verificaram que tumores mamários apresentam maior ocorrência em cadeias abdominais caudais e inguinais.

De uma maneira geral, as características macroscópicas avaliadas no experimento, exceto o tamanho, não apresentaram significância para diferenciação do tipo de tumor. Esses resultados assemelham-se aos de Rutteman e Kirpensteijn
(2003) e Lana et al. (2007), os quais consideram que a ausência desses sinais não garante a benignidade ou malignidade da lesão.

Após a realização da mastectomia e da análise histopatológica, diagnosticaram-se $40 \%$ das massas apresentando características benignas e $60 \%$ tumores malignos, conforme exposto na Tab. 2.

Tabela 2. Frequência de neoplasias mamárias em cadelas, examinadas no Setor de Obstetrícia da UNESP/Jaboticabal, segundo o diagnóstico histopatológico

\begin{tabular}{lcc}
\hline \multicolumn{1}{c}{ Classificação } & Frequência & Porcentagem (\%) \\
\hline Grupo 1 (benigno): & 24 & 40 \\
- Adenoma mamário & 4 & 6,67 \\
- Fibroadenoma & 5 & 8,33 \\
- Hiperplasia mamária & 2 & 3,33 \\
- Tecido mamário & 2 & 3,33 \\
- Tumor benigno misto & 11 & 18,33 \\
\hline Grupo 2 (maligno): & 36 & 60 \\
- Carcinoma simples tubular & 9 & 15,00 \\
- Carcinoma complexo tubular & 13 & 21,67 \\
- Carcinoma complexo túbulo papilífero & 4 & 6,67 \\
- Carcinoma células fusiformes & 1 & 1,67 \\
- Carcinoma simples sólido & 6 & 10,00 \\
- Carcinoma complexo sólido & 3 & 5,00 \\
\hline Total & 60 & 100 \\
\hline
\end{tabular}

Verificou-se que o número de tumores malignos foi superior ao de benignos, resultado que difere dos citados de Queiroga e Lopes (2002) e Carvalho (2006), que encontraram frequência equivalente para ocorrência de tumores de mama benignos e malignos em cães.

Os resultados obtidos neste estudo são importantes para estudos epidemiológicos e clínicos, pois foi verificada a frequência específica da ocorrência para cada tipo de neoplasia mamária em cadelas, segundo classificação utilizada por Misdrop et al. (1999).

Ao se avaliarem os valores semiquantitativos de VEGF nas neoplasias mamárias de cadelas, encontrou-se média de 2,22 $\pm 0,89$ para tumores malignos e 1,66 $\pm 0,91$ para benignos, com diferença para esta variável entre os grupos $(\mathrm{p}=0,0315)$. Os resultados assemelham-se aos de Graham e Myers (1999), os quais propuseram que o VEGF é um indicador do diagnóstico tumoral e do prognóstico para as neoplasias mamárias em cães.

\section{CONCLUSÕES}

Concluiu-se que as neoplasias mamárias não apresentam características de histórico reprodutivo ou exame clínico que auxiliem o diagnóstico diferencial de tumores malignos e benignos e que a histopatologia é o método conclusivo para o diagnóstico das massas mamárias, enquanto a imunoistoquímica pode ser utilizada para determinar o prognóstico da lesão.

\section{AGRADECIMENTOS}

Os autores agradecem à FAPESP pela concessão de bolsa de doutorado e pelo auxílio para a pesquisa (Processos 2008/08180-0 e 2009/51195-0). 


\section{REFERÊNCIAS}

BASTAN, A.; ÖZENÇ, E.; PIR YAGCI, I. et al. Ultrasonographic evaluation of mammary tumors in bitches. Kafkas Üniv. Vet. Fakült. Derg., v.1, p.81-86, 2009.

CARVALHO, T.B. Neoplasia mamária em cadelas: caracterização histopatológica $e$ expressão de proteínas de estresse (HSP 72). 2006. 49f. Dissertação (Mestrado) Universidade Federal de Viçosa, Viçosa.

GRAHAM, J.C.; MYERS, R.K. The prognostic significance of angiogenesis in canine mammary tumors. J. Vet. Intern. Med., v.13, p.416-418, 1999.

LANA, S.E.; RUTTEMAN, G.R.; WITHROW, S.J. Tumors of the mammary gland. In: WITHROW, S.J. e VAIL, D.M. (Eds). Withrow e MacEwen's Small Animal Clinical Oncology. 4.ed. St. Louis: Saunders Elsevier, 2007. p.619636.

LUCENA, C.E.M. Índice de avaliação ecográfica no estudo dos nódulos sólidos mamários - uma nova proposta de classificação. 2006. 152f. Tese (Doutorado) - Universidade Federal de Minas Gerais, Belo Horizonte.

MARTINS, D.C.; FERREIRA, A.M.R. Marcadores prognósticos como um auxílio à conduta clínico-cirúrgica em uma cadela apresentando múltiplos nódulos mamários. Acta Sci. Vet., v.31, p.189-191, 2003.

MISDROP, W.; ELSE, R.W.; HELLMÉN, E. et al. Histological classification of mammary tumors of the dog and the cat. In: world health organization international histological classification of tumors of domestic animals, 2ed. v.VII. Washington, DC. Armed Forces Institute of Pathology, 1999. p. 1-59.

NYMAN, H.T.; NIELSEN, O.L.; MCEVOY, F.J. et al. Comparison of B-mode and Doppler ultrasonographic findings with histologic features of benign and malignant mammary tumors in dogs. Am. J. Vet. Rese., v.67, p.985991,2006
QUEIROGA, F.; LOPES, C. Tumores mamários caninos - novas perspectivas. In: CONGRESSO DE CIÊNCIAS VETERINÁRIAS, 2002, Oeiras. Anais... Oeiras: 2002. p.183-190.

REBÊLO, A.M.G. Carcinoma ductal invasor de mama: relação da expressão imunoistoquímica do fator de crescimento do endotélio vascular (VEGF) e do oncogene BCL-2 com o tamanho tumoral e status dos linfonodos sentinelas no momento do diagnóstico histopatológico da neoplasia. 2009. 74f. Dissertação (Mestrado) Universidade de Brasília, Brasília.

RESTUCCI, B.; PAPPARELLA, S.; MAIOLINO, P. et al. Expression of vascular endothelial growth factor in canine mammary tumors. Vet. Pathol., v.39, p.488-493, 2002.

RUTTEMAN, G.R.; KIRPENSTEIJN, J. Tumours of the mammary glands. In: DOBSON, J.M. e LASCELLES, B.D.X. (Eds). BSAVA Manual of Canine and Feline Oncology. 2.ed. Gloucester: British Small Animal Veterinary Association, 2003. p.234-242.

SILVA, A.E.; SERAKIDES, R.; CASSALI, G.D. Carcinogênese hormonal e neoplasias hormônio-dependentes. Cienc. Rural, v.34, p.625-633, 2004.

TURLEY, H.; SCOTT, P.A.; WATTS, V.M. et al. Expression of VEGF in routinely fixed material using a new monoclonal antiboby VG1. J. Pathol., v.186, p.313-318, 1998.

ZUCCARI, D.A.P.C.; PAVAM, M.V.; CORDEIRO, J.A. et al. A imunoexpressão das citoqueratinas como marcadores diagnósticos e prognósticos nas neoplasias mamárias caninas. Rev. Port. Cienc. Vet., v.99, p.173-178, 2004. 